\title{
A rare case of esophageal metastasis from pancreatic ductal adenocarcinoma: a case report and literature review
}

\author{
Lauren M. Rosati ${ }^{1, *}$, Megan N. Kummerlowe ${ }^{1, *},{ }^{*}$ Justin Poling ${ }^{2}$, Amy Hacker-Prietz ${ }^{1}$, \\ Amol K. Narang ${ }^{1}$, Eun J. Shin ${ }^{3}$, Dung T. Le ${ }^{4}$, Elliot K. Fishman ${ }^{5}$, Ralph H. Hruban ${ }^{2}$, \\ Stephen C. Yang ${ }^{6}$, Matthew J. Weiss ${ }^{6}$ and Joseph M. Herman ${ }^{1,7}$ \\ ${ }^{1}$ Department of Radiation Oncology \& Molecular Radiation Sciences, The Sol Goldman Pancreatic Cancer Research Center, \\ The Johns Hopkins University School of Medicine, Baltimore, MD, USA \\ 2 Department of Pathology, The Sol Goldman Pancreatic Cancer Research Center, The Johns Hopkins University School of \\ Medicine, Baltimore, MD, USA \\ ${ }^{3}$ Department of Gastroenterology, The Johns Hopkins University School of Medicine, Baltimore, MD, USA \\ ${ }^{4}$ Department of Oncology, The Sol Goldman Pancreatic Cancer Research Center, The Johns Hopkins University School of \\ Medicine, Baltimore, MD, USA \\ ${ }^{5}$ Department of Radiology, The Sol Goldman Pancreatic Cancer Research Center, The Johns Hopkins University School of \\ Medicine, Baltimore, MD, USA \\ ${ }^{6}$ Department of Surgery, The Sol Goldman Pancreatic Cancer Research Center, The Johns Hopkins University School of \\ Medicine, Baltimore, MD, USA \\ 7 Department of Radiation Oncology, The University of Texas MD Anderson Cancer Center, Houston, TX, USA \\ * These authors have contributed equally to this manuscript
}

Correspondence to: Joseph M. Herman, email: JMHerman@mdanderson.org

Keywords: pancreatic cancer, pancreatic ductal adenocarcinoma, metastatic, esophagus, esophageal metastasis Received: April 28, $2017 \quad$ Accepted: May 20, $2017 \quad$ Published: June 12, 2017

Copyright: Rosati et al. This is an open-access article distributed under the terms of the Creative Commons Attribution License 3.0 (CC BY 3.0), which permits unrestricted use, distribution, and reproduction in any medium, provided the original author and source are credited.

\section{ABSTRACT}

Purpose: We report a very unique case of an esophageal metastasis from a pancreatic ductal adenocarcinoma (PDAC) primary.

Methods: After obtaining consent from the patient, all relevant records of the case were obtained and retrospectively reviewed.

Results: At presentation, the patient was diagnosed with synchronous pancreatic and esophageal cancer. He received six months of neoadjuvant therapy including FOLFIRINOX (5-fluorouracil, leucovorin, irinotecan, and oxaliplatin) and stereotactic body radiation therapy (SBRT) to the pancreatic tumor followed by a combined pancreaticoduodenectomy and Ivor Lewis esophagectomy. Review of the final esophageal specimen revealed normal overlying squamous mucosa with an underlying focus of metastatic PDAC. The patient remains alive with no evidence of disease 17 months from surgery and $\mathbf{2 5}$ months from diagnosis.

Conclusions: Differentiating an esophageal metastasis from a PDAC primary versus a synchronous esophageal carcinoma is very difficult despite state-of-the-art diagnostic techniques performed at a high-volume tertiary cancer center. Extensive evaluation and continued follow-up of PDAC patients presenting with a synchronous esophageal lesion in a multidisciplinary setting may help ensure efficient and accurate management. In our case, neoadjuvant FOLFIRINOX and SBRT to the primary PDAC tumor followed by surgery has been an effective approach for this patient.

\section{INTRODUCTION}

Pancreatic ductal adenocarcinoma (PDAC) is the third leading cause of death by a solid malignancy in the United States, with a 5-year overall survival rate of $8 \%$. [1] PDAC is highly aggressive and often diagnosed at an advanced stage due to the inability to detect early symptoms. An autopsy series reported that distant 
metastasis occurs late during the genetic evolution of PDAC, with an estimated half-decade required for a PDAC to acquire metastatic ability. [2]

PDAC most commonly metastasizes to lymph nodes, the liver, lung, and peritoneal cavity, while rare locations that have been reported include bone, brain, myocardium, and the umbilicus. [3, 4] At this time, there are few known cases of isolated esophageal metastasis from a pancreatic primary. In general, metastases to the esophagus are extremely rare, with rates ranging from $4-11 \%$ in patients with primaries of the lung, breast, and stomach. $[5,6]$

Not only is a PDAC metastasis to the esophagus extremely rare, but it is also difficult to distinguish an esophageal primary from a metastasis to the esophagus by radiographic imaging or endoscopy. To our knowledge, we report the $2^{\text {nd }}$ case of a metastasis to the esophagus arising from a PDAC primary reported in the modern era (since the 1980s). [7-13]

\section{RESULTS}

\section{Clinical presentation and treatment recommendations}

A 72-year-old non-smoking male presented with a 6-month history of weight loss $(9 \mathrm{~kg})$ followed by obstructive jaundice characterized by a 2 -month history of acholic stools and dark urine. Past medical history was significant for hypertension and dyslipidemia and an extensive family history of cancer was significant for pancreas, liver, breast, gynecologic, and colon malignancies in 5 siblings and his father. Initial evaluation was conducted by his primary care provider and included laboratory studies and imaging. Computed tomography (CT) scan of the abdomen and pelvis revealed a $2.5 \mathrm{x}$ $1.7 \mathrm{~cm}$ mass in the pancreatic head, abutment of the superior mesenteric artery (SMA) and vein (SMV), and marked biliary and pancreatic ductal dilatation consistent with PDAC. Liver function tests (LFTs) were elevated, with an alkaline phosphatase of $515 \mathrm{IU} / \mathrm{L}$, aspartate aminotransferase of $198 \mathrm{IU} / \mathrm{L}$, and total bilirubin of $10.3 \mathrm{mg} / \mathrm{dL}$. Carbohydrate antigen 19-9 (CA 19-9) at this time was $395 \mathrm{U} / \mathrm{mL}$. Upon further workup by a gastroenterologist, endoscopic ultrasound (EUS) with fine needle aspiration (FNA) revealed adenocarcinoma of the pancreatic head in addition to an incidental 2.0 $\mathrm{cm}$ distal esophageal exophytic lesion that returned positive for adenocarcinoma. The relationship of these two carcinomas was uncertain. Endoscopic retrograde cholangiopancreatography (ERCP) was also performed for metallic biliary stent placement to relieve high-grade biliary obstruction related to the pancreatic mass.

Further imaging with 18-fluorodeoxyglucose positron emission tomography (FDG-PET)/CT demonstrated a large hypodense mass in the head of the pancreas with moderate FDG activity consistent with the patient's known PDAC in addition to multiple enlarged peripancreatic, aortocaval, and porta hepatic lymph nodes as well as a focal area of mild metabolic activity in the distal esophagus just above the gastroesophageal junction with multiple paraesophageal lymph nodes. At an outside institution, the patient was diagnosed with localized PDAC that was thought to be unresectable along with a separate esophageal adenocarcinoma primary. Therefore, his local oncologist recommended chemotherapy with FOLFIRNOX (5-fluorouracil, leucovorin, irinotecan, and oxaliplatin) and referred the patient to our Pancreatic Multidisciplinary Clinic (PMDC) for additional recommendations. [14]

Approximately one month from initial presentation, the patient was seen in our PMDC for a second opinion. Review of the outside pathologic slides confirmed moderately differentiated adenocarcinoma of the pancreas and adenocarcinoma in the distal esophagus; however, histologic distinction of the esophageal lesion as a primary tumor or metastasis was inconclusive. A repeat CT confirmed an ill-defined 2.7 x $5.0 \mathrm{~cm}$ mass within

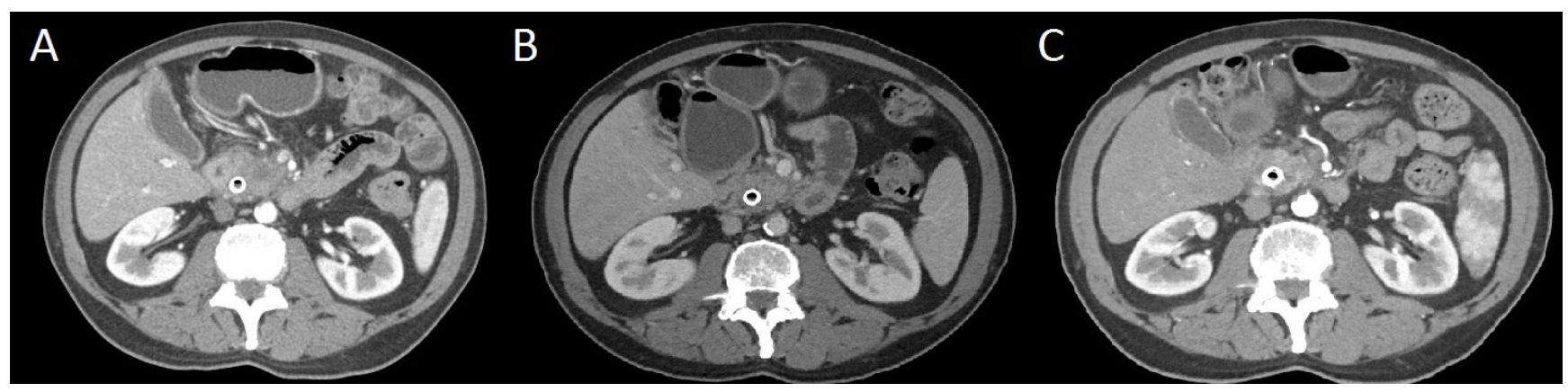

Figure 1: A. Pre-treatment computed tomography (CT) scan demonstrating ill-defined infiltrative mass measuring $2.7 \mathrm{~cm}$ x $5.0 \mathrm{~cm} . \mathbf{B}$. CT following 6 doses of FOLFIRINOX chemotherapy showing that the mass involving the head and uncinate process of the pancreas is difficult to define and measure but appears slightly less bulky as compared to the prior examination. C. 6-weeks post-SBRT CT scan reveals interval decrease in infiltrative pancreatic head mass. 
the pancreatic head/uncinate process of the pancreas invading into the $2^{\text {nd }}$ and $3^{\text {rd }}$ portions of the duodenum and demonstrating proximal main pancreatic duct dilation. Vessel involvement included encasement of the $\mathrm{SMV} /$ portal vein $(\mathrm{PV})$ confluence and $180^{\circ}$ abutment of the SMA, thereby conferring a diagnosis of borderline resectable PDAC (Figure 1A). CA 19-9 and hemoglobin A1C were elevated at this time at $315.1 \mathrm{U} / \mathrm{mL}$ and $6.4 \%$, respectively, while carcinoembryonic antigen (CEA) was within normal range $(2.4 \mathrm{ng} / \mathrm{mL})$.

Suspecting borderline resectable PDAC and an early-stage esophageal primary, our multidisciplinary team recommended neoadjuvant chemotherapy followed by standard chemoradiation (CRT) or stereotactic body radiation therapy (SBRT) with re-evaluation for potential surgical resection. FOLIFIRINOX was the recommended chemotherapy such that the platinum agent would have activity in both primary pancreatic and esophageal tumors. Depending on the expertise of the thoracic oncologists and tumor response to chemotherapy, standard CRT would be warranted in order to encompass both the esophagus and pancreas in the same field; however, if the esophageal lesion would not require neoadjuvant radiation, SBRT to the pancreas lesion would be preferred. In order to address the suspected esophageal lesion, our thoracic colleagues were consulted and the patient was referred for formal evaluation by a thoracic surgeon.

After endoscopy and thoracic surgical consultation, the esophageal lesion was thought to be a synchronous esophageal primary cancer (T1bN1Mx, with no dysphasia symptoms) and the treatment recommendation consisted of neoadjuvant FOLFIRINOX followed by SBRT and evaluation for surgery. It was understood that treatment of the PDAC was of primary significance, with the possibility of delivering definitive CRT to the esophagus later in the treatment course.

\section{Neoadjuvant therapy}

The following week, FOLFIRINOX was initiated locally and continued for 3 months (notably, irinotecan was held for the first 2 doses due to elevated LFTs). Following 6 doses of FOLFIRINOX, the patient presented back to our PMDC for re-evaluation. The patient continued to work 12-hour days throughout therapy, with his only complaint being minor fatigue. His CA 19-9 had decreased to $71.9 \mathrm{U} / \mathrm{mL}$ at this time (4 months from

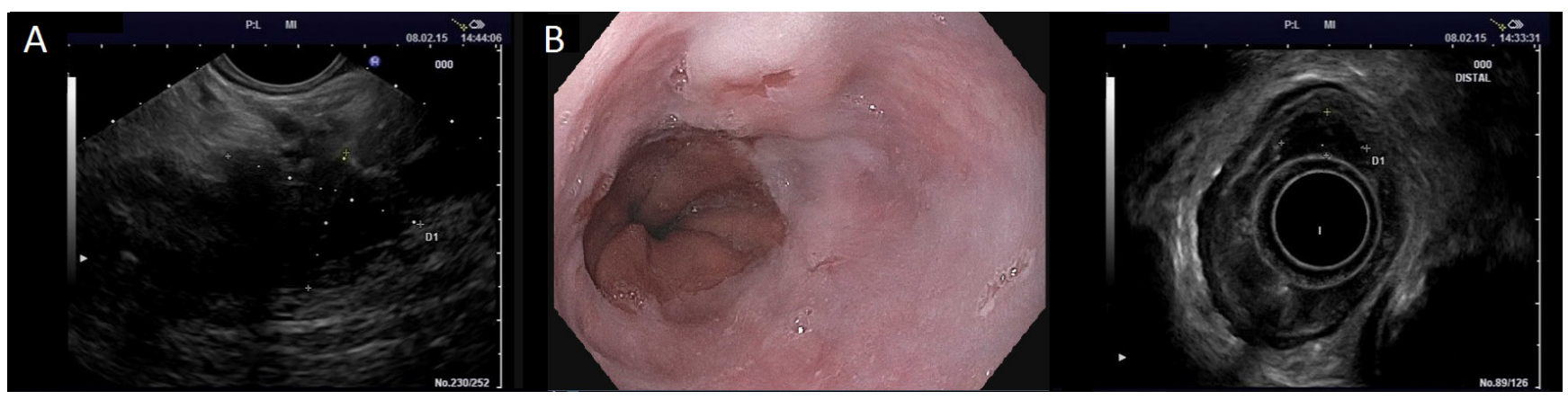

Figure 2: Visualization of the A. pancreatic lesion on endoscopic ultrasound (EUS) and B. esophageal lesion on endoscopy and EUS at the time of fiducial placement prior to SBRT.

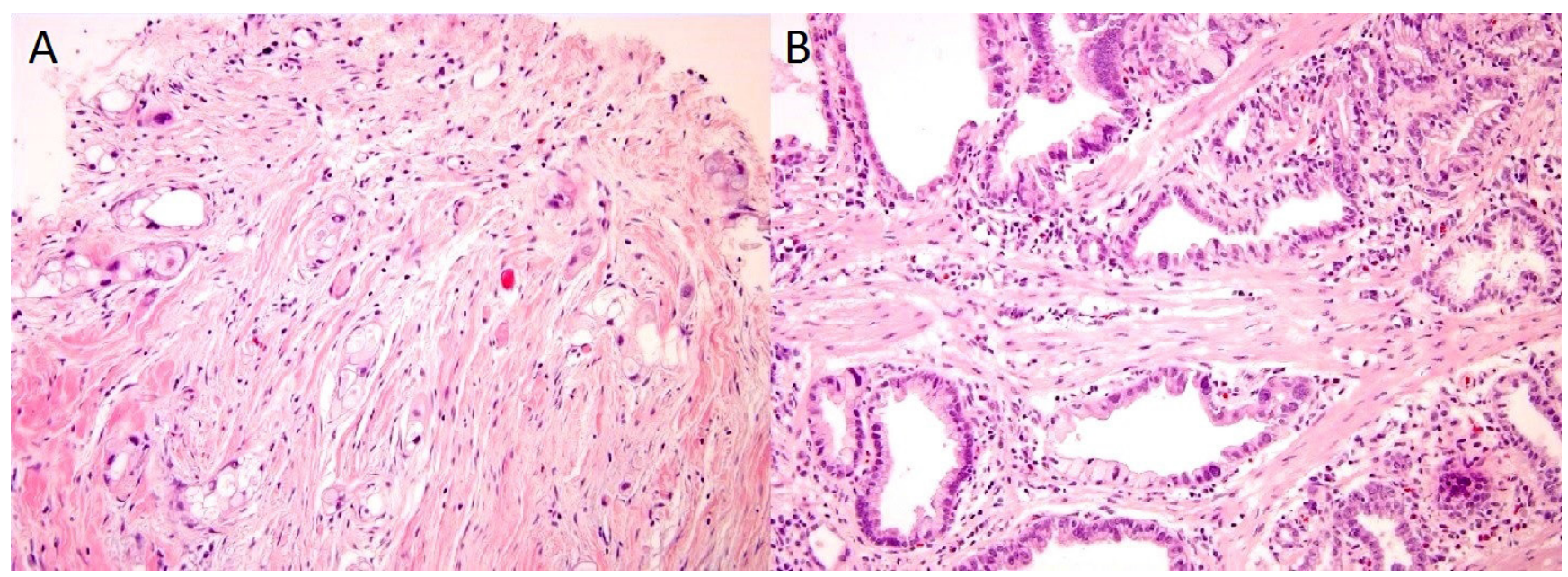

Figure 3: Evidence of fibrosis in the pancreatic primary A. and esophageal B. specimen. 
diagnosis), with CT demonstrating the pancreatic mass and regional lymphadenopathy to be slightly less bulky, improvement of SMA/SMV involvement (Figure 1B), and improved visualization of the esophageal thickening. Our multidisciplinary team recommended 2 additional months of FOLFIRINOX followed by SBRT if no disease progression and re-evaluation for surgery and/or irreversible electroporation (IRE). The patient resumed chemotherapy and received 6 additional doses, for a total of 12 doses of FOLFIRINOX over 6 months.

The patient then underwent SBRT to the pancreatic tumor to a total cumulative dose of 30.5 Gy in 5 fractions. Image guidance was performed using 3 gold fiducial markers endoscopically placed around the lesion and active breathing control (ABC) was used to minimize movement of the tumor during respiration. Images of the pancreatic and esophageal lesions at the time of endoscopy can be visualized in Figure 2. The patient's only complaint during SBRT was mild (grade 1) fatigue. Three weeks after the completion of SBRT, CT imaging showed a slight interval decrease in the infiltrative pancreatic head mass and regional lymphadenopathy without definite evidence of vascular invasion (Figure 1C). CA 19-9 further decreased to $41.7 \mathrm{U} / \mathrm{mL}$, nearly an 8-fold decrease from diagnosis. The patient was considered a surgical candidate at this time, with the plan to proceed forward with a combined approach of pancreaticoduodenectomy and esophagectomy to remove both the pancreas and esophageal tumors, respectively, in four weeks.
Of note, an esophagogastroduodenoscopy (EGD) was performed at the time of endoscopic fiducial placement to re-biopsy the esophageal lesion. The morphology was most consistent with a carcinoma that spread from the pancreaticobiliary system and immunolabeling for SMAD4 demonstrated retention of labeling, which neither confirmed nor refuted an interpretation of spread from a pancreaticobiliary lesion. The patient also experienced a few episodes of hematochezia during chemotherapy. A colonoscopy was performed and reported as negative, with the bleeding resolving spontaneously.

\section{Surgical resection}

Eight months after initial diagnosis and after six months of neoadjuvant therapy, the patient underwent a pylorus-preserving pancreaticoduodenectomy and Ivor Lewis esophagectomy with jejunostomy feeding tube (J-tube) placement. During the operation, the right gastric artery was preserved and the blood supply to the stomach was confirmed both visually and with an intraoperative Doppler ultrasound. The pancreatic specimen revealed numerous microscopic foci of adenocarcinoma with vacuolated cytoplasm and hyperchromatic nuclei scattered within a $5 \mathrm{~cm}$ fibrotic tumor bed (Figure 3A), otherwise defined as a near pathologic complete response to neoadjuvant therapy. Despite the minimal residual invasive carcinoma and extensively fibrotic background, it was considered a moderate response to neoadjuvant

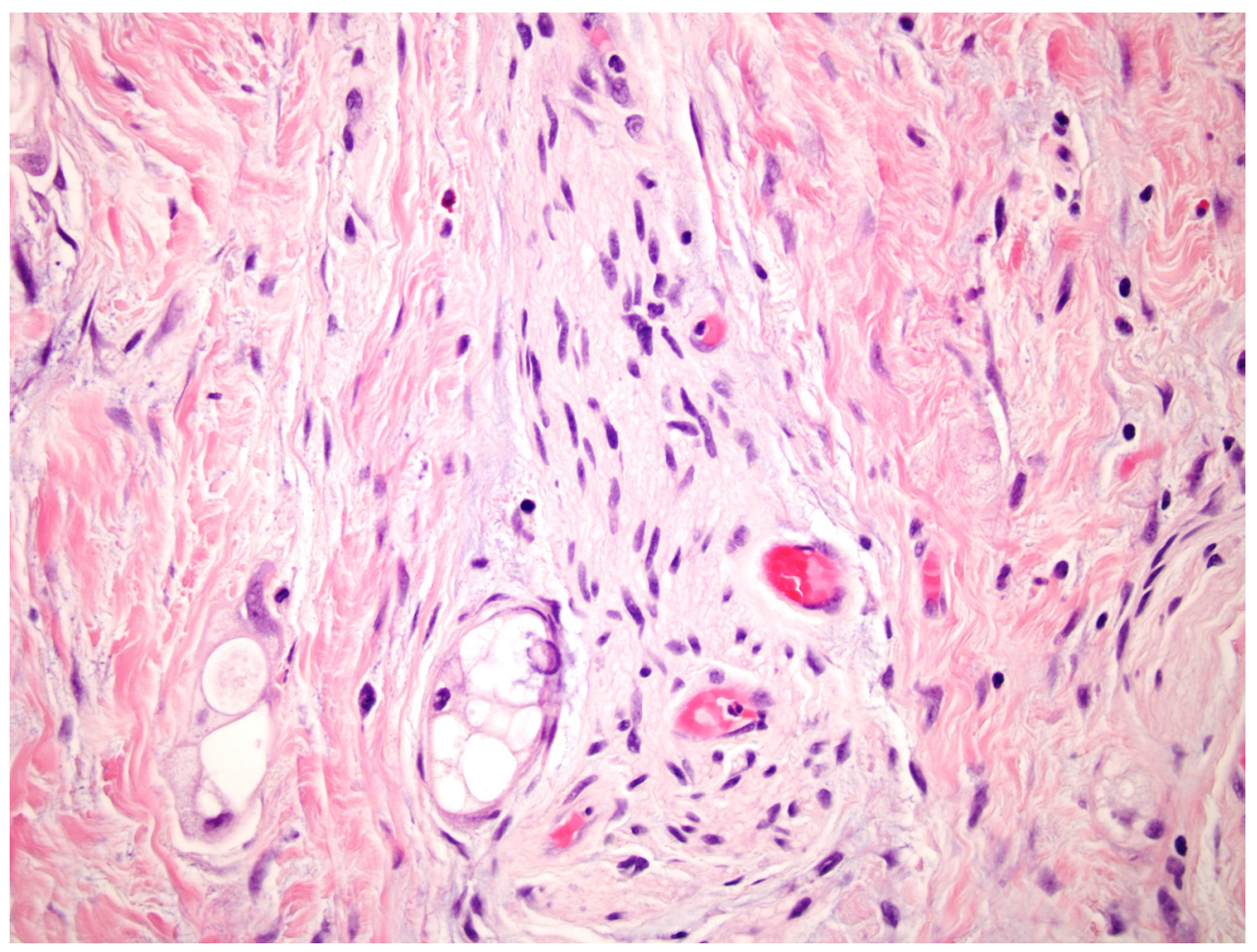

Figure 4: Evidence of perineural invasion of the pancreatic primary. 
therapy due to the number of foci present and the area across which they were dispersed. A successful marginnegative resection was achieved, with a distance of invasive carcinoma $4 \mathrm{~mm}$ to the uncinate margin; however, 3 of 16 lymph nodes contained metastatic carcinoma, with the largest tumor focus being $3 \mathrm{~mm}$. Local extension to the wall of duodenum and lymphovascular invasion were present, as was perineural invasion (Figure 4).

The esophageal specimen revealed a microscopic focus $(3 \mathrm{~mm})$ of infiltrating adenocarcinoma involving submucosa of the distal esophagus (Figure 3B) along with an incidental leiomyoma $4 \mathrm{~mm}$ in size. Resection margins were uninvolved and all 14 lymph nodes were negative for tumor. Focal Barrett mucosa of the distinctive type with Paneth cell metaplasia was observed with no evidence of high-grade dysplasia or an in situ carcinoma. Focally active chronic gastritis and chronic cholecystitis were noted. The normal overlying squamous mucosa as shown in Figure 5 is strong evidence that the esophageal lesion represents a metastasis from the patient's primary PDAC. Therefore, the final pathologic diagnosis was yT3yN1yM1 PDAC.

\section{Follow-up}

There were no major surgical complications although gram-positive cocci in chains were found in the wound and treated with antibiotics and negative-pressure wound therapy was performed for drainage at the incision site. One month from surgery, the patient was doing well with no major complaints other than intermittent abdominal pain. His J-tube was removed at the time of two month follow-up and adjuvant chemotherapy was recommended; however, numerous appointments were missed and the patient refused adjuvant therapy. Unintentional weight loss secondary to pain during eating and acid reflux led to a nutrition consultation and prescription of a proton pump inhibitor and pancreatic enzyme supplementation. The patient occasionally complains of abdominal pain and nausea but is doing well otherwise and remains with no evidence of disease 17 months from surgery and 25 months from diagnosis, with surveillance laboratory studies and imaging occurring every 3 months.

\section{DISCUSSION}

This case report summarizes a unique case of a patient with PDAC with an isolated metastasis to the esophagus who underwent successful resection of a pancreatic head and distal esophageal lesion after neoadjuvant therapy. To our knowledge, this is the $12^{\text {th }}$ reported case of an isolated esophageal metastasis from PDAC and the $2^{\text {nd }}$ report on an aggressive approach of a combined pancreaticoduodenectomy and esophagectomy.

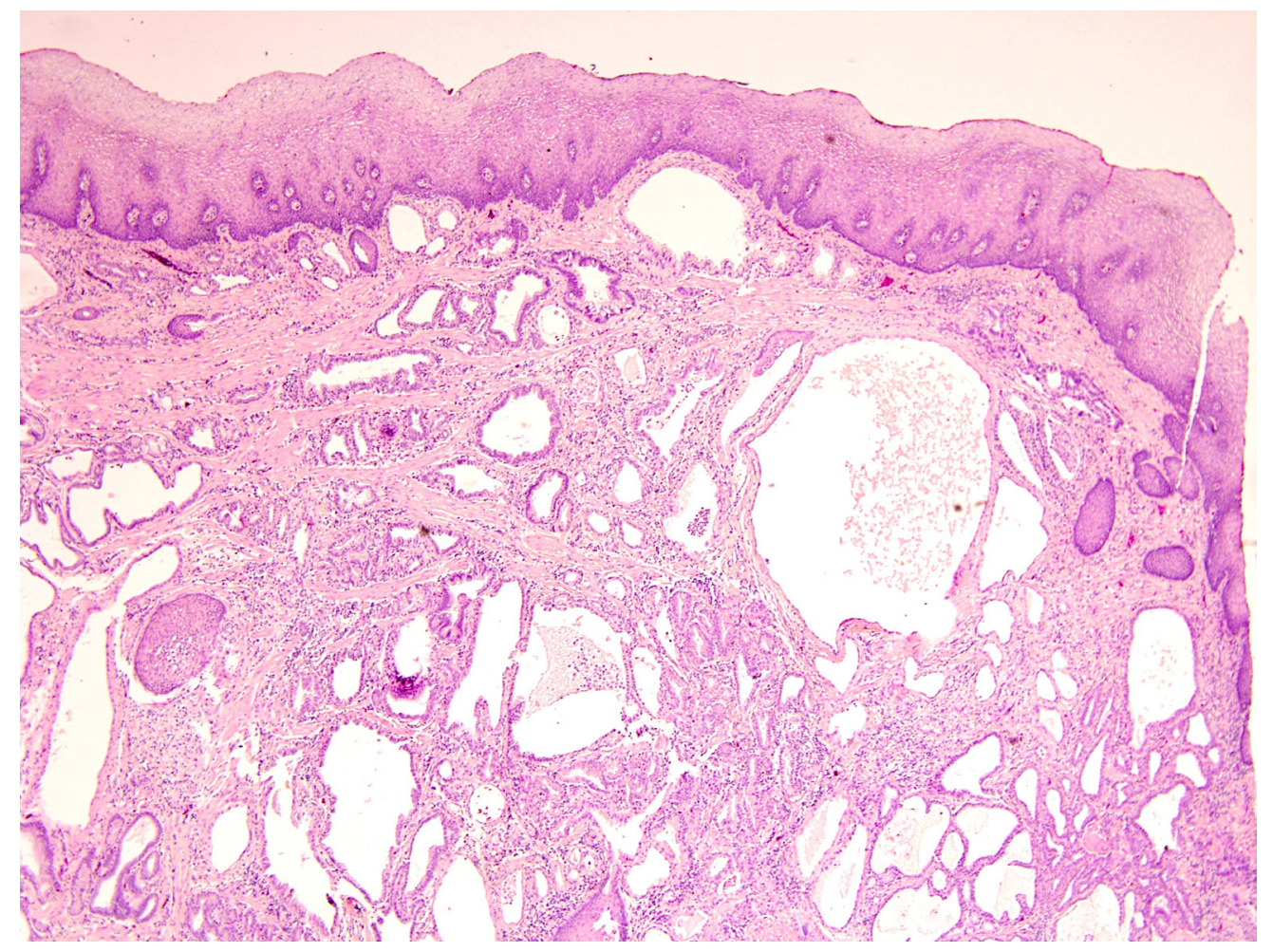

Figure 5: The lack of in situ component in the overlying epithelium provides supporting evidence that the suspected primary esophageal lesion was actually an esophageal metastasis from PDAC. 
$[7-13,15]$

The patient's pancreatic primary showed moderate treatment effect among a background of extensive fibrosis, whereas the esophageal lesion showed a more classic appearance of an untreated adenocarcinoma with numerous dilated glands with an infiltrative growth pattern, nuclear atypia, and scattered mitotic figures. While there was focal Barrett's mucosa elsewhere in the esophagus, it did not show significant dysplasia. Most significantly, the adenocarcinoma in this case had overlying squamous mucosa with no significant histopathologic change. While SMAD4 expression was intact in the esophageal lesion, roughly half of PDACs retain SMAD4 expression, and this result does not exclude spread from a PDAC primary. $[16,17]$

This case report elucidates the difficulty of differentiating an esophageal metastasis from PDAC primary versus a synchronous esophageal carcinoma. Despite numerous methods of imaging and procedures including $\mathrm{CT}$, PET/CT, EUS, EGD, and immunohistochemistry as well as multidisciplinary review among radiology, pathology, gastroenterology, surgery, medical oncology, and radiation oncology at a high-volume tertiary center, the final diagnosis of metastatic PDAC to the esophagus was not reached until a substantial amount of tissue was reviewed after partial esophagectomy. FDG-PET is commonly used in combination with $\mathrm{CT}$ and/or EUS to identify occult metastases in pancreatic and esophageal adenocarcinomas. However, the sensitivity and specificity of detection of metastases range from $50-90 \%$ and, as observed in this case report, may not lead to conclusive evidence. [18-21]

In general, local therapy is not traditionally recommended for metastatic disease, PDAC or otherwise; however, oncologists are becomingly increasingly aggressive by offering radiation therapy and/or surgical resection in this patient population, particularly in the setting of limited oligometastatic disease. [5, 22-27] In fact, surgical resection of metastases to the esophagus from distant organs has historically been reported as a promising and viable option for cases in which the primary tumor growth rate is suspected to be slow. [5] Although the morbidity and mortality associated with major operations such as a pancreatectomy and esophagectomy may be expected to be high, outcomes have improved tremendously in recent decades, especially with surgeons who are experienced and operate on a large volume of patients annually. [28, 29] Notably, combining an esophagectomy with a pancreaticoduodenectomy requires advanced planning and efficient coordination between both thoracic and hepatobiliary surgeons. A short course of radiation therapy with SBRT may also be a reasonable option to maximize local control with very little toxicity in oligometastatic PDAC, $[24,26,27,30]$ especially in cases in which a break from systemic therapy is necessary due to intolerability.
Neoadjuvant FOLFIRINOX was administered in this patient as a method to provide aggressive systemic therapy and include a platinum agent that may warrant a treatment response in both the PDAC and esophageal lesion. In 2011, Conroy and colleagues published the results of a randomized clinical trial comparing FOLFIRINOX and gemcitabine monotherapy. [31] FOLFIRINOX was superior to gemcitabine in terms of overall survival (11.1 months $v s .6 .8$ months), progressionfree survival (6.4 months vs. 3.3 months), and objective response (31.6\% vs. 9.4\%). Since then, FOLFIRINOX has been studied in other settings of PDAC as well as other gastrointestinal cancers, with promising response rates in patients who are able to tolerate the regimen. [32-35]

An esophageal metastasis from a pancreatic primary may be more common than traditional belief and patients with a suspicious esophageal lesion should undergo comprehensive evaluation and close follow-up in order to guide management. Although there are limited data to suggest an optimal approach to these cases, neoadjuvant FOLFIRNOX followed by SBRT and surgery has resulted in favorable disease control over two years from diagnosis despite no adjuvant therapy. However, we only recommend aggressive surgery of both lesions if there is no clear evidence of metastatic disease at other locations after an extended period of time ( $>6$ months). As future technologies involving novel analytic techniques such as next-generation sequencing, [36, 37] circulating tumor DNA, [38, 39] and intravital microscopy [40] advance, oncologists will be more likely to predict treatment response and make improved treatment recommendations accordingly.

\section{MATERIALS AND METHODS}

After obtaining consent from the patient, all relevant records of the case were retrospectively reviewed. The pathologic specimens were reviewed with response to neoadjuvant therapy graded using the criteria described previously by the College of American Pathologists. [41]

\section{Abbreviations}

Pancreatic ductal adenocarcinoma (PDAC); Computed tomography (CT); Superior mesenteric artery (SMA); Superior mesenteric vein (SMV); Liver function tests (LFTs); Carbohydrate antigen 19-9 (CA 19-9); Endoscopic ultrasound (EUS); Fine needle aspiration (FNA); Endoscopic retrograde cholangiopancreatography (ERCP); 18-fluorodeoxyglucose positron emission tomography (FDG-PET); FOLFIRINOX (5-fluorouracil, leucovorin, irinotecan, and oxaliplatin); Pancreatic Multidisciplinary Clinic (PMDC); Portal vein (PV); Carcinoembryonic antigen (CEA); Chemoradiation (CRT); Stereotactic body radiation therapy (SBRT); Irreversible 
electroporation (IRE); Active breathing control (ABC); Esophagogastroduodenoscopy (EGD); Jejunostomy feeding tube (J-tube)

\section{Author contributions}

Lauren M. Rosati: Miss Rosati contributed to the conceptualization and methodology development of the project. She performed a literature review and data collection to present, write, and revise the proposed manuscript. She also managed coordination and communication with the patient and among co-authors.

Megan N. Kummerlowe: Miss Kummerlowe contributed to the literature review, data collection, writing, revising, and presenting the proposed manuscript. She also maintained communication with the patient.

Justin Poling: Dr. Poling analyzed the pathologic features of the resected specimen. He also contributed to the writing and revision of the proposed manuscript.

Amy Hacker-Prietz: Mrs. Hacker-Prietz provided her expertise in radiation oncology in treating the patient and assisting with preparation and proofreading of the proposed manuscript.

Amol K. Narang: Dr. Narang provided his expertise in radiation oncology in treating the patient and assisting with preparation and proofreading of the proposed manuscript.

Eun J. Shin: Dr. Shin provided her expertise in gastroenterology in treating the patient and assisting with preparation and proofreading of the proposed manuscript and presentation of the figures.

Dung T. Le: Dr. Le provided her expertise in medical oncology in treating the patient and assisting with preparation and revision of the proposed manuscript.

Elliott K. Fishman: Dr. Fishman oversaw the review of radiographic imaging and assisting with preparation and proofreading of the proposed manuscript.

Ralph H. Hruban: Dr. Hruban oversaw the pathologic review of the case and assisting with preparation and proofreading of the proposed manuscript.

Stephen C. Yang: Dr. Yang provided his expertise in thoracic surgery and contributed to the preparation and revision of the proposed manuscript.

Matthew J. Weiss: Dr. Weiss provided his expertise in pancreaticobiliary surgery and contributed to the writing and proofreading of the proposed manuscript.

Joseph M. Herman: Dr. Herman provided his expertise in radiation oncology in treating the patient and contributed to the conceptualization, methodology development, writing, and revision of the proposed manuscript. He supervised the project in its entirety.

\section{CONFLICTS OF INTEREST}

None.

\section{CONSENT}

Verbal consent was obtained from the patient for publication of this case report and accompanying images. Institutional guidelines regarding the publication of a case report were followed.

\section{FUNDING}

Viragh Family Foundation, Claudio X. Gonzalez Family Foundation, Keeling Family Foundation, DeSanti Family Foundation, McKnight Family Foundation

\section{REFERENCES}

1. Siegel RL, Miller KD, Jemal A. Cancer Statistics, 2017. CA Cancer J Clin. 2017; 67: 7-30.

2. Yachida S, Jones S, Bozic I, Antal T, Leary R, Fu B, Kamiyama M, Hruban RH, Eshleman JR, Nowak MA, Velculescu VE, Kinzler KW, Vogelstein B, et al. Distant metastasis occurs late during the genetic evolution of pancreatic cancer. Nature. 2010; 467: 1114-1117.

3. Robinson BW, Lewis RR. Myocardial metastasis from carcinoma of pancreas presenting as acute myocardial infarction. Journal of the Royal Society of Medicine. 1982; 75: $560-562$.

4. Crescentini F, Deutsch F, Sobrado CW, Araujo S. Umbilical mass as the sole presenting symptom of pancreatic cancer: a case report. Revista do Hospital das Clinicas. 2004; 59: 198-202.

5. Mizobuchi S, Tachimori $\mathrm{Y}$, Kato H, Watanabe H, Nakanishi Y, Ochiai A. Metastatic esophageal tumors from distant primary lesions: report of three esophagectomies and study of 1835 autopsy cases. Japanese journal of clinical oncology. 1997; 27: 410-414.

6. Simchuk EJ, Low DE. Direct esophageal metastasis from a distant primary tumor is a submucosal process: a review of six cases. Diseases of the esophagus. 2001; 14: 247-250.

7. Toreson WE. Secondary carcinoma of the esophagus as a cause of dysphagia. Arch Pathol. 1944; 38: 82-84.

8. Pygott F. Radiological appearances in pancreatic cancer. The British journal of radiology. 1950; 23: 656-666.

9. Langton L, Laws JW. Dysphagia in carcinoma of the pancreas. J Fac Radiol. 1954; 6: 134-138.

10. Ward P. Pulmonary and Oesophageal Presentations of Pancreatic Carcinoma. The British journal of radiology. 1964; 37: 27-33.

11. Fisher MS. Metastasis to the esophagus. Gastrointestinal radiology. 1976; 1: 249-251.

12. Anderson MF, Harell GS. Secondary esophageal tumors. AJR Am J Roentgenol. 1980; 135: 1243-1246.

13. Murakami T, Elquza E, Ozden N, Pugh J. A rare finding of direct esophageal metastasis from a pancreatic primary tumor. Pancreas. 2014; 43: 1123-1125. 
14. Pawlik TM, Laheru D, Hruban RH, Coleman J, Wolfgang CL, Campbell K, Ali S, Fishman EK, Schulick RD, Herman JM, and Johns Hopkins Multidisciplinary Pancreas Clinic Team. Evaluating the impact of a single-day multidisciplinary clinic on the management of pancreatic cancer. Annals of surgical oncology. 2008; 15: 2081-2088.

15. Tobari S, Ikeda Y, Kurihara H, Takami H, Okinaga K, Kodaira S. Effective treatment with chemotherapy and surgery for advanced small cell carcinoma of the esophagus. Hepato-gastroenterology. 2004; 51: 1027-1029.

16. Tascilar M, Offerhaus GJ, Altink R, Argani P, Sohn TA, Yeo CJ, Cameron JL, Goggins M, Hruban RH, Wilentz RE. Immunohistochemical labeling for the Dpc4 gene product is a specific marker for adenocarcinoma in biopsy specimens of the pancreas and bile duct. American Journal of Clinical Pathology. 2001; 116: 831-837.

17. Iacobuzio-Donahue CA, Fu B, Yachida S, Luo M, Abe H, Henderson CM, Vilardell F, Wang Z, Keller JW, Banerjee P, Herman JM, Cameron JL, Yeo CJ, et al. DPC4 gene status of the primary carcinoma correlates with patterns of failure in patients with pancreatic cancer. Journal of clinical oncology. 2009; 27: 1806-1813.

18. Sihvo EI, Rasanen JV, Knuuti MJ, Minn HR, Luostarinen ME, Viljanen T, Farkkila MA, Salo JA. Adenocarcinoma of the esophagus and the esophagogastric junction: positron emission tomography improves staging and prediction of survival in distant but not in locoregional disease. Journal of gastrointestinal surgery. 2004; 8: 988-996.

19. van Westreenen HL, Westerterp M, Bossuyt PM, Pruim J, Sloof GW, van Lanschot JJ, Groen H, Plukker JT. Systematic review of the staging performance of $18 \mathrm{~F}$-fluorodeoxyglucose positron emission tomography in esophageal cancer. Journal of clinical oncology. 2004; 22: 3805-3812.

20. Farma JM, Santillan AA, Melis M, Walters J, Belinc D, Chen DT, Eikman EA, Malafa M. PET/CT fusion scan enhances CT staging in patients with pancreatic neoplasms. Annals of surgical oncology. 2008; 15: 2465-2471.

21. Gabriel E, Alnaji R, Du W, Attwood K, Kukar M, Hochwald S. Effectiveness of Repeat 18F-Fluorodeoxyglucose Positron Emission Tomography Computerized Tomography (PET-CT) Scan in Identifying Interval Metastases for Patients with Esophageal Cancer. Annals of surgical oncology. 2017; 24:1739-1746.

22. Simmonds PC, Primrose JN, Colquitt JL, Garden OJ, Poston GJ, Rees M. Surgical resection of hepatic metastases from colorectal cancer: a systematic review of published studies. British journal of cancer. 2006; 94: 982-999.

23. Kanas GP, Taylor A, Primrose JN, Langeberg WJ, Kelsh MA, Mowat FS, Alexander DD, Choti MA, Poston G. Survival after liver resection in metastatic colorectal cancer: review and meta-analysis of prognostic factors. Clinical epidemiology. 2012; 4: 283-301.

24. Herman JM, Hoffman JP, Thayer SP, Wolff RA. Management of the Primary Tumor and Limited Metastases in Patients With Metastatic Pancreatic Cancer. Journal of the National Comprehensive Cancer Network. 2015; 13: e29-36.

25. Balzano G, Capretti G, Callea G, Cantu E, Carle F, Pezzilli R. Overuse of surgery in patients with pancreatic cancer. A nationwide analysis in Italy. HPB. 2016; 18: 470-478.

26. Lischalk JW, Burke A, Chew J, Elledge C, Gurka M, Marshall J, Pishvaian M, Collins S, Unger K. FiveFraction Stereotactic Body Radiation Therapy (SBRT) and Chemotherapy for the Local Management of Metastatic Pancreatic Cancer. Journal of gastrointestinal cancer. 2017.

27. Redmond KJ, Lo SS, Dagan R, Poon I, Foote MC, Erler D, Lee Y, Lohr F, Biswas T, Ricardi U, Sahgal A. A multinational report of technical factors on stereotactic body radiotherapy for oligometastases. Future oncology (London, England). 2017; 13:1081-1089.

28. Bouras G, Burns EM, Howell AM, Bagnall NM, Lee H, Athanasiou T, Darzi A. Systematic review of the impact of surgical harm on quality of life after general and gastrointestinal surgery. Annals of Surgery. 2014; 260: 975983.

29. Mamidanna R, Ni Z, Anderson O, Spiegelhalter SD, Bottle A, Aylin P, Faiz O, Hanna GB. Surgeon Volume and Cancer Esophagectomy, Gastrectomy, and Pancreatectomy: A Population-based Study in England. Annals of Surgery. 2016; 263: 727-732.

30. Rosati LM, Herman JM. Role of Stereotactic Body Radiotherapy in the Treatment of Elderly and Poor Performance Status Patients With Pancreatic Cancer. Journal of oncology practice. 2017; 13: 157-166.

31. Conroy T, Desseigne F, Ychou M, Bouche O, Guimbaud R, Becouarn Y, Adenis A, Raoul JL, Gourgou-Bourgade S, de la Fouchardiere C, Bennouna J, Bachet JB, KhemissaAkouz $\mathrm{F}$, et al. FOLFIRINOX versus gemcitabine for metastatic pancreatic cancer. The New England journal of medicine. 2011; 364: 1817-1825.

32. Ychou M, Viret F, Kramar A, Desseigne F, Mitry E, Guimbaud R, Delpero JR, Rivoire M, Quenet F, Portier G, Nordlinger B. Tritherapy with fluorouracil/leucovorin, irinotecan and oxaliplatin (FOLFIRINOX): a phase II study in colorectal cancer patients with non-resectable liver metastases. Cancer chemotherapy and pharmacology. 2008; 62: 195-201.

33. Moorcraft SY, Khan K, Peckitt C, Watkins D, Rao S, Cunningham D, Chau I. FOLFIRINOX for locally advanced or metastatic pancreatic ductal adenocarcinoma: the Royal Marsden experience. Clinical colorectal cancer. 2014; 13: 232-238.

34. Paniccia A, Edil BH, Schulick RD, Byers JT, Meguid C, Gajdos C, McCarter MD. Neoadjuvant FOLFIRINOX application in borderline resectable pancreatic adenocarcinoma: a retrospective cohort study. Medicine. 2014; 93: e198.

35. Lockhart AC, Krajewski KA, Wang-Gillam A, Amin 
M, Sorscher S, Lim KH, Tan BR, Picus J, Hecky A, Allen K, Peterson JD, O'Day E, de Wilton Marsh R, et al. FOLFIRINOX as first-line therapy in patients with metastatic gastroesophageal cancers (GEC). J Clin Oncol. 2015 (Suppl 3); 33:177.

36. Kyrochristos ID, Glantzounis GK, Ziogas DE, Gizas I, Schizas D, Lykoudis EG, Felekouras E, Machairas A, Katsios C, Liakakos T, Cho WC, Roukos DH. From Clinical Standards to Translating Next-Generation Sequencing Research into Patient Care Improvement for Hepatobiliary and Pancreatic Cancers. International journal of molecular sciences. 2017.

37. Makohon-Moore AP, Zhang M, Reiter JG, Bozic I, Allen B, Kundu D, Chatterjee K, Wong F, Jiao Y, Kohutek ZA, Hong J, Attiyeh M, Javier B, et al. Limited heterogeneity of known driver gene mutations among the metastases of individual patients with pancreatic cancer. Nature genetics. 2017; 49: 358-366.

38. Cheng H, Liu C, Jiang J, Luo G, Lu Y, Jin K, Guo M, Zhang Z, Xu J, Liu L, Ni Q, Yu X. Analysis of ctDNA to predict prognosis and monitor treatment responses in metastatic pancreatic cancer patients. International journal of cancer. 2017; 140:2344-2350.
39. Poruk KE, Blackford AL, Weiss MJ, Cameron JL, He J, Goggins MG, Rasheed Z, Wolfgang CL, Wood LD. Circulating Tumor Cells Expressing Markers of Tumor Initiating Cells Predict Poor Survival and Cancer Recurrence in Patients with Pancreatic Ductal Adenocarcinoma. Clinical cancer research. 2017; 23:26812690.

40. Beerling E, Oosterom I, Voest E, Lolkema M, van Rheenen J. Intravital characterization of tumor cell migration in pancreatic cancer. Intravital. 2016; 5: e1261773.

41. Washington K, Berlin J, Branton P, Burgart LJ, Carter DK, Compton CC, Fitzgibbons P, Frankel WL, Jessup J, Kakar S, Minsky B, Nakhleh R, Tang LH. Protocol for the Examination of Specimens From Patients With Carcinoma of the Pancreas. College of American Pathologists. Northfield, IL. 2016. Available from: http:/www.cap.org/ ShowProperty?nodePath=/UCMCon/Contribution Folders/ WebContent/pdf/cp-pancreasexo-16protocol-3400.pdf. 